\title{
Chief Cell
}

National Cancer Institute

\section{Source}

National Cancer Institute. Chief Cell. NCI Thesaurus. Code C12580.

One of three cell types that are found in the gastric or parathyroid glands, or in the carotid body. Gastric chief cells secrete pepsinogen and chymosin. Parathyroid chief cells secrete parathyroid hormone. Type 1 (chief) cells in the carotid body contain neurosecretory vesicles that may play a role in chemoreception. 